\title{
TWO INTEGRATED EXPERIMENTAL AND MODELING APPROACHES TO STUDY STRAIN DISTRIBUTIONS IN NICKEL AND NICKEL-BASE SUPERALLOY POLYCRYSTALS
}

\author{
T.J. Turner ${ }^{a}$, P.A. Shade ${ }^{a}$, J. Schuren ${ }^{a}$, M.A. Groeber ${ }^{\mathrm{a}}$, M. Miller ${ }^{\mathrm{b}}$, M.D. Uchic ${ }^{\mathrm{a}}$ \\ ${ }^{a}$ Air Force Research Laboratory, Materials and Manufacturing Directorate, AFRL/RXLMP, \\ 2230 Tenth Street, Wright-Patterson, AFB, OH 45433-7817, USA \\ ${ }^{b}$ Sibley School of Mechanical and Aerospace Engineering \\ Cornell University, Rhodes Hall, Ithaca, NY 14850, USA
}

Keywords: Crystal-Plasticity, Synchrotron, X-Ray Diffraction, Digital Image Correlation, Lattice Strain

\begin{abstract}
This work presents two integrated experimental and modeling approaches for examining polycrystals at different length scales, which utilize different experimental techniques but the same elasto-crystal plasticity based finite element model (CPFEM). The goal of this work is to calibrate modeling approaches through experimental data, and then use the models to gain insight into the mechanics of deformation in nickel and nickel-base superalloy polycrystals. The first study utilized a micro-tensile test specimen of pure nickel with 259 grains, where the CPFEM simulations were initiated with the explicit 3D microstructure as measured with 3D-Electron Back Scattering Detection (EBSD) serial sectioning and compared with surface deformations measured with Digital Image Correlation (DIC). The second study utilized a larger polycrystalline nickel-base superalloy specimen with approximately 50,000 grains, where the CPFEM simulation results are compared with lattice strain data obtained through high energy $\mathrm{x}$-ray diffraction utilizing a synchrotron $\mathrm{x}$-ray source. In both cases the simulations are compared with different aspects of the experimental strain information (surface strain or lattice strain), and then the simulations are used to explore aspects of the heterogeneous nature of the deformation that are difficult or impossible to measure experimentally.
\end{abstract}

\section{Introduction}

The strategic development of new engineering materials with improved performance rests on establishing computational models that link materials processing, microstructure, and properties/performance. The greater scientific community has long sought these linkages, but without access to experimental data at the relevant length scales, validated models capable of predicting complex phenomena such as fatigue initiation and small-crack propagation have remained elusive. Without validated models, adoption of advanced computational methods will remain at the mercy of traditional large-scale testing programs which inherently limit the adoption of new materials or innovative designs due to fiscal and time constraints. Therefore, the development of experimental methods capable of validating materials models over the appropriate processing or service conditions - i.e., length/time scales, deformation/temperature regimes - are essential to the rapid design, development, and deployment of novel materials, or current materials in new applications.

In this work, we make direct comparisons of simulation results with experimental measurements in an attempt to calibrate an
Elasto-Crystal Plasticity Finite Element Model (CPFEM) for use in investigating complex deformation phenomena. In particular, we developed two distinct CPFEM simulations utilizing the same material model, both coupled with relevant physical experiments that capture the deformation of nickel and nickel-base superalloy materials at two different length scales. The goal is to calibrate the modeling methodology and then utilize the simulation results to gain insight into the physics of deformation for these materials. Eventually, we hope to understand the sources of non-uniform deformation and the onset of failure in nickel-base alloy systems such that critical aerospace components can be designed and improved.

Many researchers have strived to compare simulation results to experiments. Some studies compare bulk crystallographic texture changes with deformation, while others examine local lattice orientation changes and the development of intra-granular misorientation and deformation [1-8]. While those studies are useful in setting the groundwork for this study, they often stop after validating their particular model and methodology. For this work we go beyond a simple model calibration and validation, and apply our model to study aspects of the deformation that cannot be experimentally measured. From this we hope to gain insight into the mechanics of deformation, as well as to understand where experimental techniques may fall short in order to develop new relevant experiments for the future.

The work presented here builds upon the techniques presented in [9], where microstructural features at the relevant length-scales were experimentally characterized and used to develop a CPFEM model that incorporated aspects of the microstructure. The model was then validated by direct comparison to other experimental data, and additional simulations were performed to examine the effect of sub-surface grains on surface strain distributions, as well as the difference in deformation partitioning between surface and subsurface grains.

\section{Materials and Experimental Procedures}

\section{$\underline{2.1 \text { Material }}$}

For this work we examined two different materials: commercially pure nickel foil and Low Solvus High Refractory (LSHR) nickelbase superalloy. The pure nickel foil material was annealed and had a purity of $99.0 \%$, and was purchased commercially with a foil thickness of $50 \mu \mathrm{m}$. Although not shown, Electron BackScatter Diffraction (EBSD) measurements revealed a uniform 
distribution crystallographic orientations and an approximate average equiaxed grain size of $20 \mu \mathrm{m}$.

The LSHR material had the chemistry shown in Table 1 [10], and was heat treated at $1135^{\circ} \mathrm{C}$ for 3 hours with an oil quench to produce a fine grained microstructure with an approximate average grain size of $15 \mu \mathrm{m}$ as determined through EBSD analysis of the material (not shown).

Table 1. Composition of LSHR in weight \% - balance nickel

\begin{tabular}{|l|l|l|l|l|l|l|l|l|l|l|}
\hline Co & $\mathrm{Cr}$ & $\mathrm{Al}$ & $\mathrm{Ti}$ & $\mathrm{W}$ & $\mathrm{Mo}$ & $\mathrm{Ta}$ & $\mathrm{Nb}$ & $\mathrm{C}$ & $\mathrm{B}$ & $\mathrm{Zr}$ \\
\hline 21 & 13 & 3.5 & 3.5 & 4.3 & 2.7 & 1.6 & 1.5 & .03 & .03 & .05 \\
\hline
\end{tabular}

\subsection{Micro-Tension Test on Nickel Foil}

Micro-tensile samples were fabricated from the nickel foil by implementing a stencil mask technique. This technique involves standard microelectronics processing methods to produce high aspect-ratio patterned templates, i.e. stencil masks, from a Si wafer. Once fabricated, the stencil masks are placed on top of a pre-thinned metallic substrate and the mask-and-substrate are cosputtered using a broad ion beam milling system which acts to transfer the 2D pattern of the stencil mask onto the metallic substrate. The final sample geometry was defined using focused ion beam (FIB) milling through an automated process that serially milled the perimeter surface of each sample while maintaining a back-tilt of $1^{\circ}$ to ensure orthogonal sidewalls. The fabricated tensile specimen consisted of a rectangular cross section, a gage width of $21 \mu \mathrm{m}$, a thickness of $38 \mu \mathrm{m}$, and a gage length of $80 \mu \mathrm{m}$. The FIB was also used to machine a grid of points onto the top surface of the samples to be used as fiducial markers for digital image correlation (DIC) tracking of surface displacements. The points were circular with a diameter of $30 \mathrm{~nm}$ and a point-to-point spacing of $2.3 \mu \mathrm{m}$. An image of one of the samples prior to testing is shown in Figure 1.

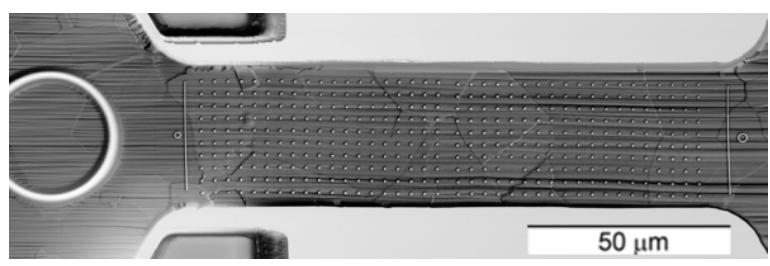

Figure 1. Pure nickel micro-tension test specimen

In-situ SEM-based mechanical testing was conducted using a custom-built mechanical testing device [11-13]. Forces were applied with a piezoelectric actuator, loads were measured with a strain-gage-based load cell, and displacements were calculated from image data by tracking the positional change of two surface markers along the gage length. An alignment fixture ensures linear motion of the loading train and the samples are precisely positioned for testing by attaching the bulk substrate to a piezoelectric controlled $x-y-z$ positioning stage. The specimen was deformed nominally in tension along the gage length to an axial true strain of $2.4 \%$. Due to challenges in mechanical testing at this length-scale, the loading end of the fixture was allowed to move laterally during the deformation in an attempt to self-align the specimen while under load. Examination of the deformed sample geometry indicates that the specimen did deform laterally at the loaded end, indicating that the applied deformation was not a perfect uni-axial tension test. Throughout the deformation the location of the fiducial markers on the specimen surface was tracked through automated SEM imaging to derive the surface strain distribution.

\subsection{Serial Sectioning - Microstructure Statistics of Nickel Foil}

After the tensile test, the full 3D microstructure and internal crystallographic lattice rotations of the deformed sample were characterized through destructive 3D-EBSD serial sectioning using custom automation software and an FEI Nova 600 DB FIBSEM equipped with a TSL Hikari high speed EBSD detector. Cross-sections were repeatedly milled with a section thickness of $250 \mathrm{~nm}$, while crystallographic orientation data was captured on each section through integrated EBSD collection using a $20 \mathrm{keV}$ electron beam and a pixel size of $250 \mathrm{~nm}$. The 3D-EBSD dataset consisted of approximately 400 sections and required 5-6 days of collection time. The full 3D post-deformation microstructure was subsequently re-constructed using DREAM 3-D software (http://dream3d.bluequartz.net/). Internal lattice rotations were removed and the structure was meshed, resulting in the input data for the CPFEM model. The re-constructed microstructure revealed 259 individual grains within the gage section of the specimen. Although not shown, the measured crystallographic texture was essentially random in nature.

\subsection{Lattice Strain Pole Figure (SPF) Experiment on LSHR}

In the lattice Strain Pole Figure (SPF) experiment, an LSHR tensile specimen with a nominal gage section of $8 \mathrm{~mm} \times 1 \mathrm{~mm} \times$ $1 \mathrm{~mm}$ was loaded under uni-axial tension while in situ high-energy $\mathrm{x}$-ray diffraction measurements were performed to monitor the distortion of the crystals (nominally 50,000 grains) during the deformation [14-18]. The high-energy diffraction experiments were performed at the Cornell High-Energy Synchrotron Source (CHESS). During loading the specimen was reoriented relative to the $x$-ray beam in order to interrogate every crystal within the diffraction volume (the volume of the specimen that intersects the beam-approximately $500 \mu \mathrm{m}$ x $500 \mu \mathrm{m} \times 1 \mathrm{~mm})$. Following the convention in [18], the sample was rotated about two orthogonal axes with $\omega$ ranging between -29 to 39 degrees and $\chi$ from 0 to 22 degrees in 11 increments. Lattice strains - which can be thought of as similar to the normal strains indicated by a resistance strain gage during elastic deformation, manifest themselves as shifts in diffraction peaks calculated from the diffraction data. Lattice strains were measured in many directions and used to generate lattice SPFs. Previous efforts have shown that there are appreciable variations in the average stress for different crystallographic orientations within a polycrystal even when subjected to simple uni-axial tension $[17,19]$.

The specimen was loaded to failure at approximately $12 \%$ axial true strain, although for this study we focus on data collected in the first 3\% axial true strain, closely corresponding with the micro-tension test. At specified load increments during the deformation (400MPa, 800MPa, \& $1200 \mathrm{MPa}$ ), the specimen was unloaded by $10 \%$ - to minimize material evolution due to creep [20]—while high-energy diffraction measurements were performed. The measurements involved rotating the specimen and taking multiple diffraction images, necessitating the $10 \%$ unloading as the measurements took approximately 1 hour of collection time. 


\section{Modeling Approach}

To model the uni-axial tension deformation of both the nickel micro-tension specimen and the LSHR tensile specimen, we utilized an elasto-crystal-plasticity formulation coupled with a finite-element-method model-CPFEM. The initial microstructure of the micro-tension specimen was determined through serial section data, while the initial microstructure of the LSHR specimen was characterized by EBSD with the crystallographic orientations sampled from the high-energy diffraction data. The details of the simulations, the crystalplasticity model, and the creation of the virtual digital microstructures from the EBSD scans are all non-trivial and are discussed below.

\section{$\underline{\text { 3.1 Polycrystal Model }}$}

The crystal-plasticity model employed for this work was a continuum slip model that approximates the micro-mechanics of plastic flow in crystalline solids. It consists of an anisotropic elastic response, a constitutive model written at the single crystal level, and a finite element method that assembles the single crystal responses into the overall response of the polycrystalline aggregate. The crystallographic texture, coupled with the anisotropic response of single crystals, is assumed to be the primary source of plastic anisotropy. A brief overview of the single crystal constitutive model is presented here; a more detailed development may be found in [5-7, 21-22].

The salient features of the crystal plasticity model for this work include the elastic formulation, the constitutive formulation and the hardening equation. The crystal elasticity is assumed to be linearly anisotropic, and in the case of small elastic strains, it is expressed as:

$$
\bar{\tau}=L \bar{\varepsilon}^{*}
$$

where $\boldsymbol{L}$ is the elasticity tensor, $\bar{\tau}$ is the Kirchhoff stress, and $\bar{\varepsilon}^{*}$ is the elastic strain tensor. $\boldsymbol{L}$ is expressed in terms of the anisotropic elastic constants $\left(\mathrm{c}_{11}, \mathrm{c}_{12}, \mathrm{c}_{44}\right)$ for cubic materials [23]. The rate of shearing on each slip system is determined from a rate-dependent constitutive formulation relating the shearing rate to the resolved shear stress:

$$
\dot{\gamma}^{\alpha}=\Phi\left(\tau^{\alpha}, g^{\alpha}\right)=\dot{\gamma}_{o}\left|\frac{\tau^{\alpha}}{g^{\alpha}}\right|^{1 / m} \operatorname{sgn}\left(\tau^{\alpha}\right)
$$

in which $\dot{\gamma}^{\alpha}$ denotes the rate of shearing on the $\alpha^{\text {th }}$ slip system , $\tau^{\alpha}$ and $g^{\alpha}$ are the resolved shear stress and slip-system strength of the $\alpha^{\text {th }}$ slip system, respectively, $m$ is the rate-sensitivity, and $\dot{\gamma}_{o}$ is a model parameter. The slip-system strengths are evolved through a Voce-type saturation law [24-27] of the following form:

$$
\dot{g}^{\alpha}=\theta(\dot{\gamma}, g)=h_{0}\left(\frac{g_{s o}-g^{\alpha}}{g_{s o}-h_{s}}\right) \dot{\gamma}
$$

Here, $\dot{\gamma}$ is the net rate of slip on all the slip systems within the crystal, and $h_{o}, g_{s 0}$, and $h_{s}$ are material parameters.
This model is a modification of that proposed by Asaro et. al, [2831], but does not include the effects of latent hardening. We apply a simplification to the hardening law in equation 3 , whereby we use a unique slip-system strength in each $\operatorname{crystal}\left(g^{\alpha}=g\right)$, explicitly enforcing the constraint that every slip-system in the crystal will have the same slip-system strength. This is also known as Taylor hardening [32-33], and is a common assumption used in other research, particularly when examining large strain deformations [1-3, 5-7, 8]. We chose not to include latent hardening without experimental evidence in our material system of how hardening on one slip-system affects the other slipsystems in each crystal.

\subsection{Finite Element Model - Virtual Specimens}

The collective response of the aggregate forms the macroscopic response in the polycrystalline model. In this case, the macroscopic velocity gradient is partitioned among the individual crystals through the explicit decomposition that arises via the finite element method [5-7]. Using a finite element model ensures compatibility through the choice of element shape functions, and equilibrium is satisfied in the usual finite element weak sense. The constitutive response of the material was determined with the single-crystal model discussed above, and was coupled with the Arbitrary Lagrangian/Eulerian 3D (ALE3D) finite-element code developed at Lawrence Livermore National Laboratory [34]. We employed an individual crystal orientation for each finite element, and unique grains in the model were then defined by a collection of elements with the same/similar orientation. This technique has been used in other efforts to produce virtual microstructures for CPFEM simulations [8-9].

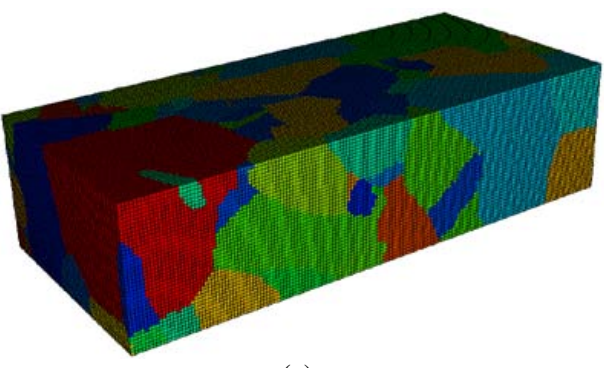

(a).

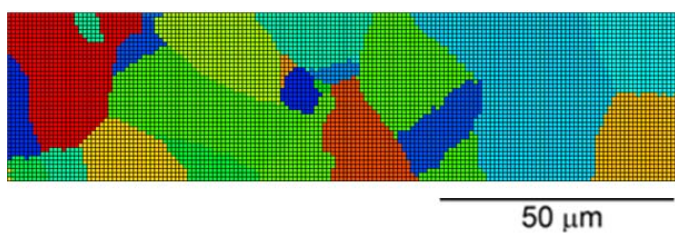

(b)

Figure 2. a. CPFEM model of the micro-tension specimen, b. the side of the CPFEM model that matches the micro-tension specimen of Figure 1

3.2.1 Micro-Tension Virtual Specimen For the micro-tension specimen we created a CPFEM model of the gage length using 448,000 8-noded hexahedral elements with an 8-point quadrature rule. This model explicitly represents the $3 \mathrm{D}$ microstructure determined from the serial section data, where the individual lattice orientation measurements and their correlated spatial locations were used to instantiate the model. Lattice rotations due to deformation in the experiment were removed by assigning the 
grain average crystallographic orientation to all of the finite elements which compose an individual grain. In this manner we assumed the starting grains were all uniform in their crystallographic orientation with no spatial variation across the grain. Figure 2a shows the CPFEM model representing the 259 grains in the cross-section of the micro-tension specimen, where the color of each grain is correlated to crystallographic orientation. Figure 2b. shows the side of the CPFEM which matched the side of the micro-tension specimen where the DIC was recorded utilizing the fidicuial markers as seen in Figure 1.

3.2.2 High-Energy Diffraction Virtual Specimen For the highenergy diffraction experiment we simulated the approximate 500 $\mu \mathrm{m}$ x $500 \mu \mathrm{m}$ x $1 \mathrm{~mm}$ diffraction volume utilizing 432,000 finite elements, where each grain was represented by a cube of 2 x 2 x 2 elements (54,000 grains). The grains are defined by a common crystallographic orientation sampled from a random crystallographic distribution. The grains were therefore cubic in shape, arranged in a regular grid, and of equal initial size. This model does not retain the fidelity of the microstructure as in the micro-tension specimen, but allows us to simulate the response of a much larger number of grains. Figure 3 shows the CPFEM model for the high-energy diffraction experiments, where once again the color of each grain is correlated to crystallographic orientation.

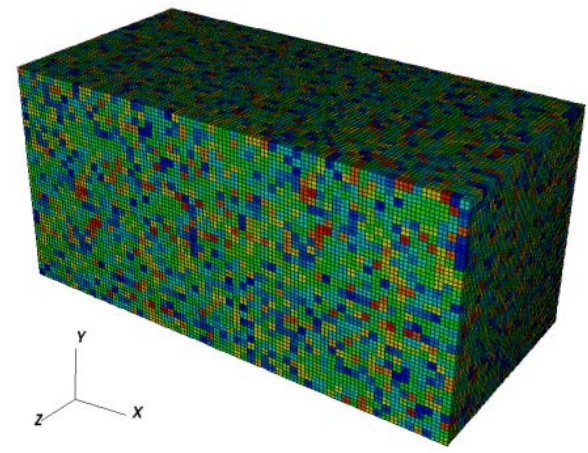

Figure 3. CPFEM model of the high-energy diffraction experiment using 54,000 grains approximating a volume of $1 \mathrm{~mm} \times 0.5 \mathrm{~mm} \times 0.5 \mathrm{~mm}$

\subsection{Simulations}

For both the micro-tension specimen and the high-energy diffraction specimen, the crystal plasticity model parameters $\left(h_{o}\right.$,

$g_{s 0}, h_{s}, g_{\text {initial }}^{\alpha}, \dot{\gamma}_{0}$, and $m$ ) were initialized such that the macroscopic stress-strain response from the CPFEM simulations matched the macroscopic stress-strain response of the experiments. In addition, the standard anisotropic elastic moduli $\left(c_{11}, c_{12}, c_{44}\right)$ were chosen to match the elastic response of the experiments, and slip was assumed to only occur on the octahedral family of $\{111\}<110>$.

3.3.1 Micro-Tension Test In the simulations for the micromechanical test we modeled the entire $21 \mu \mathrm{m}$ x $38 \mu \mathrm{m}$ x $80 \mu \mathrm{m}$ gage section and applied boundary conditions analogous to the experimental test, where one end was fixed and the other end allowed to extend and deflect laterally. Again, this is not a perfect uni-axial tension deformation, but the model boundary conditions were chosen to best match the experiment.

The crystal plasticity model parameters are shown in Table 2 below. The elastic constants were chosen to match the elastic portion of the macroscopic stress-strain response $\left(c_{11}=247 \mathrm{GPa}\right.$, $\left.\mathrm{c}_{12}=147 \mathrm{GPa}, \mathrm{c}_{44}=125 \mathrm{GPa}\right)$.

Table 2. Material Parameters for the Simulation of the micro-tension test

\begin{tabular}{|l|c|c|c|c|c|}
\hline$h_{o}$ & $g_{\text {so }}$ & $h_{s}$ & $g_{\text {initial }}^{\alpha}$ & $\dot{\gamma}_{0}$ & $m$ \\
\hline$(\mathrm{MPa})$ & $(\mathrm{MPa})$ & $(\mathrm{MPa})$ & $(\mathrm{MPa})$ & $\left(\mathrm{s}^{-1}\right)$ & - \\
\hline 500 & 103 & 43 & 43 & $1 \mathrm{e}^{-5}$ & 0.06 \\
\hline
\end{tabular}

3.3.2 High-Energy Diffraction Simulation For the high-energy diffraction specimen, we simulated a volume that contained 54,000 grains, closely matching the number of grains measured experimentally. The model was deformed in uni-axial tension until the axial true stress reached $1200 \mathrm{MPa}$, and then the simulation was unloaded by $10 \%$ to $1080 \mathrm{MPa}$, analogous to the experiment. The material parameters were calibrated such that the macroscopic stress strain from the simulation matched that of the experiment. In addition, at the $1080 \mathrm{MPa}$ load point, the lattice strains were extracted from the simulation data. In the experiment, multiple diffraction measurements at different load increments were performed to determine the evolution of lattice strain throughout the loading, but for the sake of brevity we compare only the lattice strains at the $1080 \mathrm{MPa}$ load level, as that best corresponds to the same overall true strain level seen in the mircotension test.

The model parameters used in the simulation of the diffraction specimen are shown in Table 2 . The single crystal elastic constants were $\mathrm{c}_{11}=250.74 \mathrm{GPa}, \mathrm{c}_{12}=189.6 \mathrm{GPa}, \mathrm{c}_{44}=157.2 \mathrm{GPa}$.

Table 3. Material Parameters for the Simulation of the diffraction test

\begin{tabular}{|l|c|c|c|c|c|}
\hline$h_{o}$ & $g_{\text {so }}$ & $h_{s}$ & $g_{\text {initial }}^{\alpha}$ & $\dot{\gamma}_{0}$ & $m$ \\
\hline$(\mathrm{MPa})$ & $(\mathrm{MPa})$ & $(\mathrm{MPa})$ & $(\mathrm{MPa})$ & $\left(\mathrm{s}^{-1}\right)$ & - \\
\hline 500 & 910 & 182.5 & 365 & $1 \mathrm{e}^{-5}$ & 0.06 \\
\hline
\end{tabular}

\section{Results}

\subsection{Experimental Observations}

The two experiments-one on the pure-nickel foil micro-tension specimen and the other on the larger aggregate high-energy diffraction LSHR superalloy specimen-both evaluate strain distributions in the material. However, in the micro-tension specimen we consider the strain on one surface as measured through DIC, while in the high-energy diffraction specimen we evaluate lattice strains from the diffracted volume in the bulk of the material.

4.1.1 Micro-Tension Test By tracking the fiducial markers on the micro-tension specimen, we calculated the surface strain distributions for the specimen throughout the deformation. Figure 4 shows the axial and transverse components of the strain for the specimen after unloading from peak load during the deformation. Even though the average axial true strain for the entire specimen 
was only $2.4 \%$, selected regions of the material deformed to several times that level. The heterogeneous nature of the deformation is apparent in Figure 4.
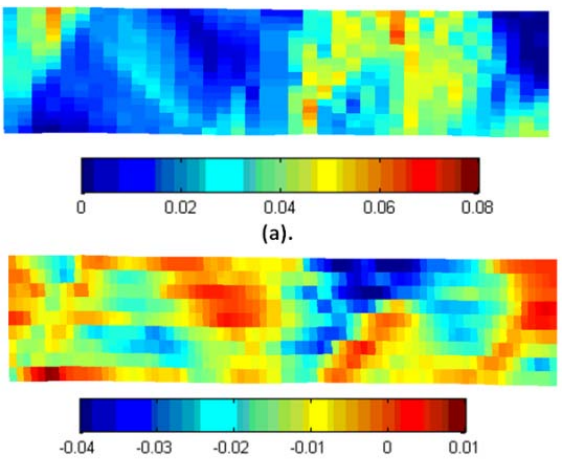

(b)

Figure 4. Experimental micro-tension specimen (a). axial strain component (b). transverse strain component

4.1.2 Lattice Strain Pole Figure (SPF) Experiment The results for the SPF experiments are shown in the first three pole figures at the top of Figure 5-which includes $\{111\},\{200\}$ and $\{220\}$ SPFs, where the loading direction is along the 1-axis. These SPFs were evaluated from the experimental diffraction data at an axial stress of $1080 \mathrm{MPa}(10 \%$ unloaded form a maximum axial stress of $1200 \mathrm{MPa}$ ) More details about the formation of the SPF from the diffraction data can be found in Schuren et al. and Miller et al. $[18,35]$. The data presented in Figure 5 represent in situ lattice strain measurements and show that the largest lattice strains for each $\{h k l\}$ develop along the tensile loading direction. Compressive strains develop along the transverse directions due to the Poisson effect. We can also see that the $\{200\}$ SPF has the largest magnitude in lattice strains, while both the $\{111\}$ and $\{220\}$ directions appear to have similar values.

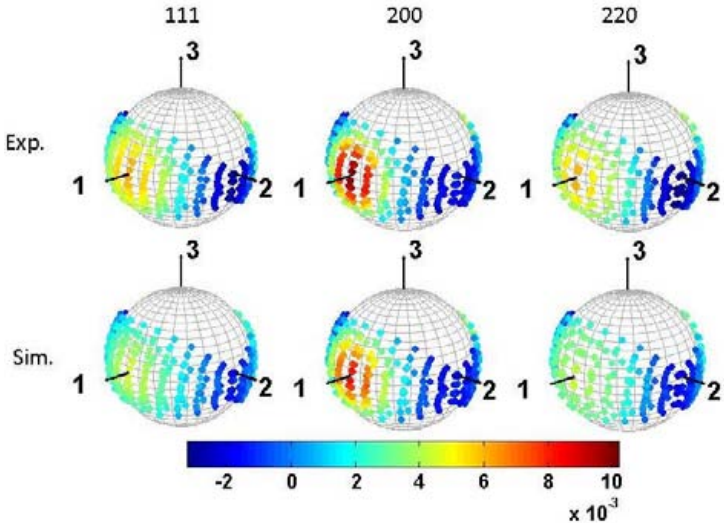

Figure 5. SPFs for the $\{111\},\{200\}$ and $\{220\}$ lattice directions, with experimental SPFs on the top row and simulation SPFs on the bottom row for comparison

\section{$\underline{4.2 \text { Simulation Results }}$}

Simulation results for both the micro-tension specimen and the high-energy diffraction specimen were evaluated in an analogous fashion to the experiments. Therefore the micro-tension simulation compared individual grain-level strain distributions on a single surface, while in the SPF diffraction experiments we compared lattice strains averaged from the diffracting volume, not simply strains on the surface.

4.2.1 Micro-Tension Test For the micro-tension simulation we evaluated the strain on the same surface as the DIC measurements from the experimental specimen. In this case we used the location of the surface nodes from the finite element mesh as the analog of the fiducial markers from the experiment. This provided a higher fidelity strain resolution since the finite element mesh was more finely distributed across the surface than the fiducial markers on the experimental micro-tension specimen. Figure 6 shows the axial and transverse components of the strain for the simulation in the unloaded state after reaching $2.4 \%$ axial true strain.

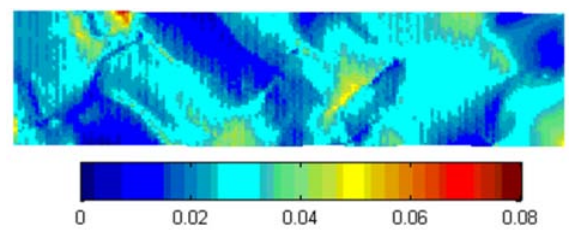

(a).

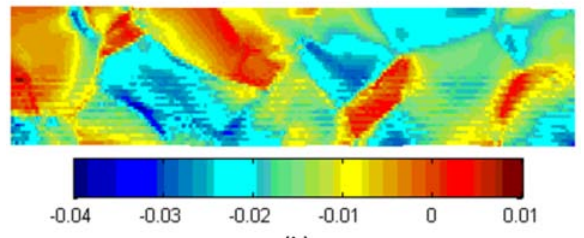

(b).

Figure 6. CPFEM simulation of micro-tension specimen (a). axial strain component, (b). transverse strain component

4.2.2 High-Energy Diffraction Simulation For the high-energy diffraction simulation we evaluated the distribution in lattice strain by simulating 54,000 crystals deformed under uni-axial tension to $3.0 \%$ axial true strain. Figure 7 shows the axial stress component plotted in the deformed simulation at the $1200 \mathrm{MPa}$ load level, including slices into the interior of the mesh. From this figure we can see that the heterogeneity is extensive, with some grains experiencing over $1 \mathrm{GPa}$ stress in the axial direction, while others see as little as 360MPa.

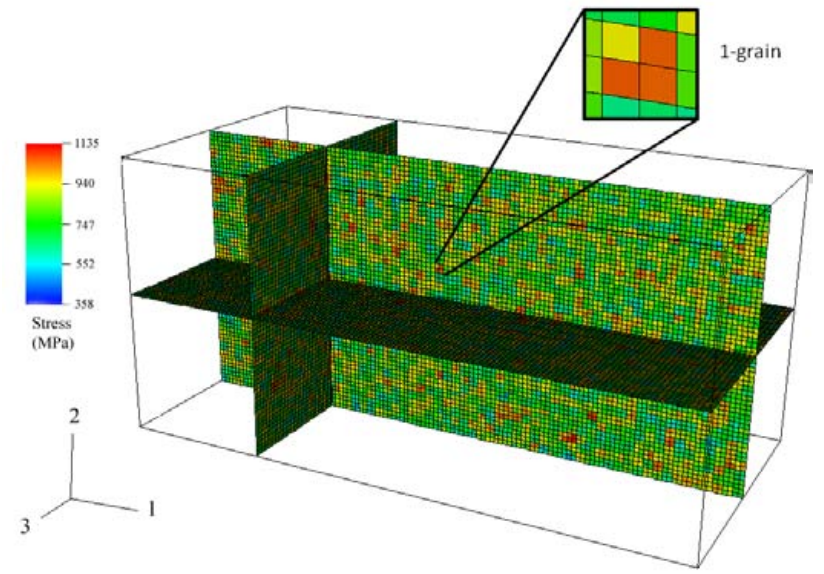

Figure 7. Axial stress $\left(\sigma_{11}\right)$ component plotted on slices through the CPFEM model after $3.0 \%$ axial true strain $(1080 \mathrm{MPa})$ 
From this simulation we were able to extract the lattice strains for each grain after unloading $10 \%$ from the peak load of $1200 \mathrm{MPa}$ average axial stress [36]. Using those lattice strains and the crystallographic orientation of the grain, we constructed SPFs analogous to those from the experiment. The bottom row of SPFs in Figure 5 show the results of the simulated diffraction experiment, with simulated SPFs for the $\{111\},\{200\}$ and $\{220\}$ lattice directions. The match between the experimental and simulated SPFs and the comparison of the macroscopic stress indicate a strong comparison between experiment and simulation.

\subsection{Influence of Sub-Surface Neighborhood on Micro-Tension}

In addition to a comparison between the experimental and simulation results (i.e. comparing Figure 4 to Figure 6), we used the CPFEM model to further examine the influence of grain neighborhood on the deformation of the micro-tension specimen. Specifically, we wanted to study the effect of crystallographic orientation in the sub-surface grains on the surface strain distribution-where we define sub-surface as any grain that does not touch the surface where the DIC measurements are evaluated. To do this, we maintained the morphology of the entire specimen constant, i.e. the grain shapes, sizes, and locations. We also kept the crystallographic orientation of the surface grains the same as the original simulation, while changing the crystallographic orientations of the grains below the DIC surface. Since the texture of the nickel foil was approximately random, we exchanged the original orientations of the sub-surface grains for new orientations sampled from a random crystallographic orientation distribution.

4.3.1 Simulations with Random Neighborhoods We randomly permuted the sub-surface grains 10 times, running simulations of the 448,000 element CPFEM models to the full $2.4 \%$ axial strain deformation for each permutation. Then we calculated the surface strain distribution for each permutation. Figure 8 shows a representative axial and transverse strain distribution from one of the permutations as an example.

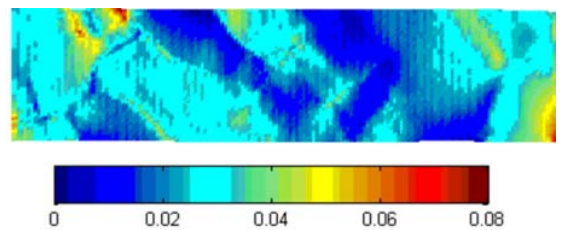

(a).

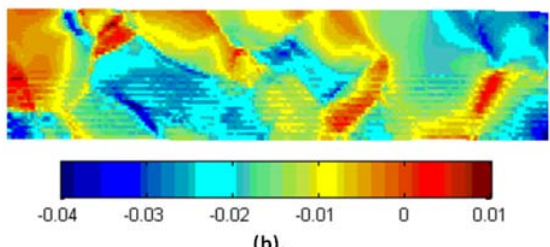

(b).

Figure 8. Representative CPFEM simulation of micro-tension specimen with permuted sub-grain crystallographic orientations (a). axial strain component, (b). transverse strain component

Although there are differences between Figure 8 and the original simulation in Figure 6, it is hard to compare the figures directly and understand the location and magnitudes of those differences in relation to the original microstructure. Therefore, to show the effect of the sub-surface crystallographic orientation, we plotted the normalized difference in the strain components between the permuted surface strain maps $\left(\varepsilon_{n n}\right)$ and the original CPFEM simulation of the surface strain $\left(\varepsilon^{0}{ }_{n n}\right)$-where $n n$ is either the axial strain component $\left(\varepsilon_{11}\right)$ or the transverse strain $\left(\varepsilon_{22}\right)$ and is evaluated at each nodal point in the mesh. Figures 9 and 10 show the normalized difference maps in the axial and transverse strain components, respectively, for three different permuted cases. These figures were calculated through the use of equation 4 , which defines a norm operation for the comparison for both the axial and transverse strain components at each nodal point in the mesh. Figure 9 shows examples of this normalized difference for the axial component of strain for three different permuted simulations, while Figure 10 uses the same permutations but displays the results for the transverse strain component.

$$
d_{n n}=\sqrt{\left(\varepsilon_{n n}-\varepsilon_{n n}^{0}\right)^{2}}
$$
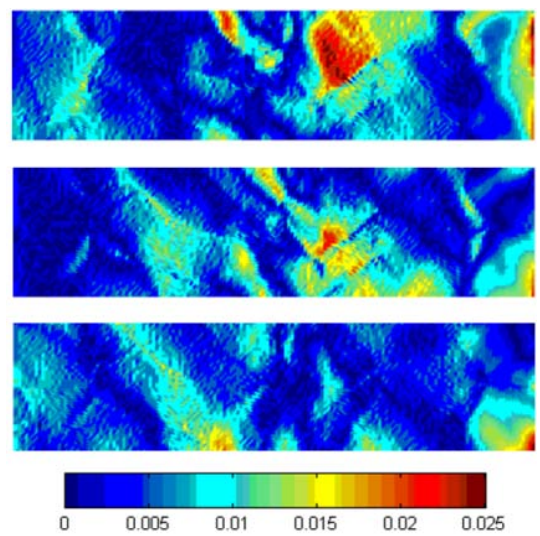

Figure 9. Axial strain $\left(\varepsilon_{11}\right)$ differences for three different CPFEM simulations with permuted sub-grain crystallographic orientations as compared to the explicit microstructure simulation from Fig. 6A
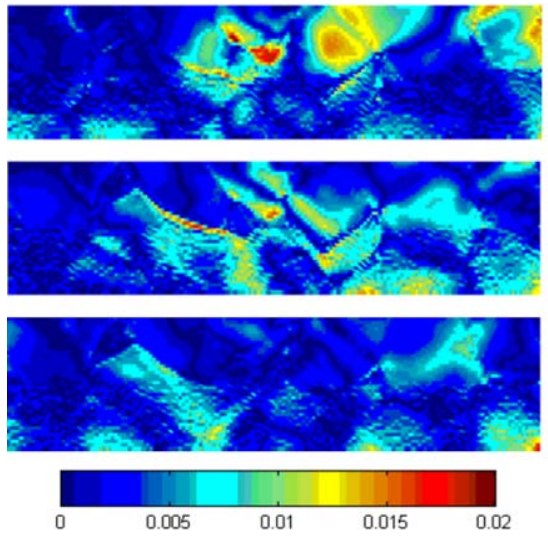

Figure 10. Transverse strain $\left(\varepsilon_{22}\right)$ differences for three different CPFEM simulations with permuted sub-grain crystallographic orientations as compared to the explicit microstructure simulation from Fig. 6B 


\subsection{Influence of Surface Grains}

In an analogous fashion to the micro-tension simulations, we use the simulated diffraction specimen to gain insight into the heterogeneous deformation in the material. In particular, we examined the difference in stress states that exist on the surface of the specimen as contrasted with the interior of the specimen. This is partly to understand the limitations of using surface only DIC measurements to measure the response of polycrystalline ensembles. After calibrating the simulation of the diffraction specimen through comparisons of the SPFs, we evaluated the difference in stress state for grains at the surface of the specimen and contrasted it with the state of stress for grains which were entirely within the interior. In this analysis with the particular simulation geometry chosen in Figure 3, there are 6728 surface grains and 45,472 grains in the interior of the specimen. Grains on either end along the $\mathrm{x}$-axis where boundary conditions were applied to the model were discarded from the analysis.

To evaluate the state of stress we took the components of the stress tensor and wrote them as a 6-component vector [37-39] as in equation 5, where $\bar{\sigma}$ is the stress vector written in terms of the components of the stress tensor relative to the macroscopic or laboratory coordinate system.

$$
\bar{\sigma}=\left[\sigma_{x x}, \sigma_{y y}, \sigma_{z z}, \sigma_{x y}, \sigma_{x z}, \sigma_{y z}\right]
$$

In the case of pure uni-axial tension, where the $\mathrm{x}$-direction is parallel to the macroscopic tensile direction, only the $\sigma_{x x}$ component is non-zero $\left(\bar{\sigma}_{\text {axial }}=\left[\sigma_{x x}, 0,0,0,0,0\right]\right)$. Therefore we evaluated the stress state in each grain by comparing the vectorized-stress in the grain with the idealized stress from the uni-axial tension test. The coaxiality of the different stress states is determined as an angle between the two vectors through the usual dot-product operation [39].

$$
\Theta=a \cos \left(\frac{\bar{\sigma}_{\text {axial }} \cdot \bar{\sigma}}{\left|\bar{\sigma}_{\text {axial }}\right||\bar{\sigma}|}\right)
$$

This angle provides a scalar measure of how much the stress state in each grain varies from the macroscopic uni-axial applied stress. Figure 11 shows the results of this analysis where the bulk-interior and the surface grains are plotted separately as a probability distribution function.

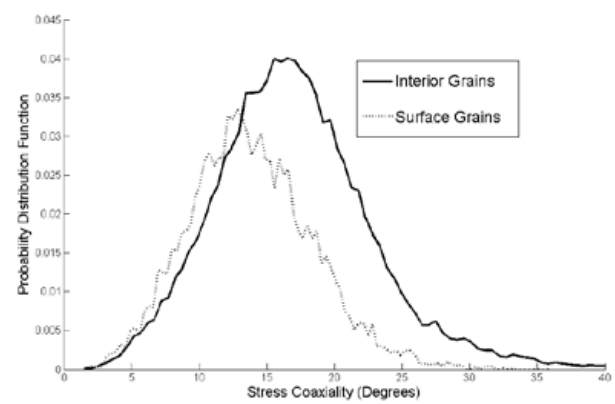

Figure 11 . The stress coaxiality for the surface and interior grains in the diffraction specimen simulation
In addition to Figure 11, we can also plot the normalized magnitude of the stress vector $\left(|\bar{\sigma}| /\left|\bar{\sigma}_{\text {axial }}\right|\right)$ in each grain, which provides even more insight into the stress distribution throughout the polycrystalline aggregate. Figure 12 shows the stress coaxiality plotted against the normalized stress magnitude, where each point in the plot (red for interior grains or blue for surface grains) represents the stress state of one grain in the aggregate. In this plot a grain experiencing a perfect uni-axial tension stress state would have a stress coaxiality of $\Theta=0$, and a normalized stress magnitude of 1.0. This is shown in Figure 12 as the black ' $x$ ' along the $y$-axis. As evident in this figure, no grains in the aggregate experience uni-axial tension, although the stress state of the entire specimen averages out to this condition. This will be discussed further in the subsequent section.

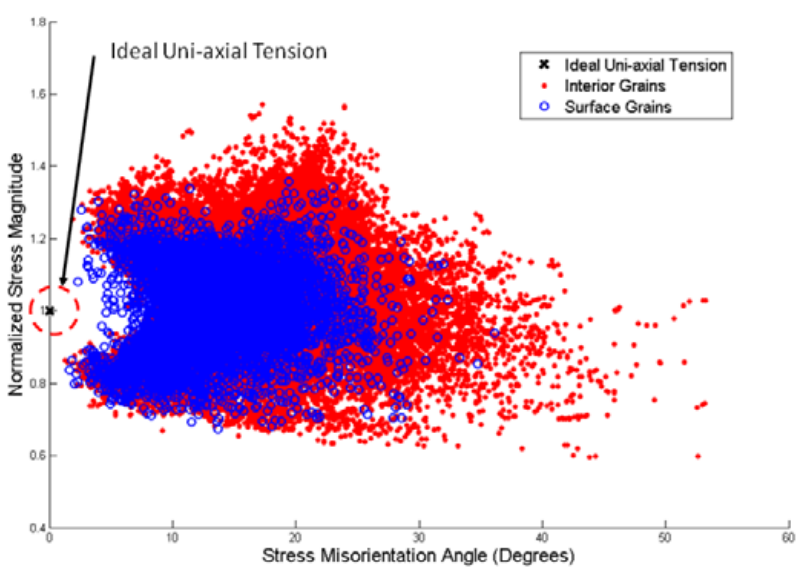

Figure 12. Plot of stress coaxiality against stress magnitude for both the surface and the interior grains in the diffraction specimen simulation

\section{Discussion}

Our objective in the present work was to examine the deformation of nickel and nickel-base materials at different length scales, and then develop a modeling methodology to gain insight into the deformation of these materials. The experimental observations and accompanying CPFEM simulations of the uni-axial tension of both the pure nickel foil and the LSHR nickel-base superalloy shed light on deformation heterogeneity exhibited under a relatively simple loading condition. We expect much more complicated stress states to exist in an arbitrary aerospace component manufactured from these materials. Therefore, it is important to understand the sources of heterogeneity and their implication on the performance and ultimate life of the material. In both of the cases presented in this work, the CPFEM models were compared with experimental observations and the models were subsequently utilized to explore the sources of heterogeneous deformation that are not easily characterized through experimental techniques alone.

For the micro-tension test there are several points worthy of discussion that will help interpret the results. Figure 4 shows both the axial and transverse strain components for the experimental micro-tension test determined from the DIC measurements. Significant heterogeneity exists throughout the surface of the specimen. This heterogeneity would be better captured had the 
fiducial markers been less coarse. In some grains on the surface of the micro-tension specimen, only a few markers span the length of individual grain, as seen in Figure 1. Clearly with the amount of heterogeneity seen in Figure 4, the scale of the fiducial markers needs to be reduced to enhance the fidelity of the DIC measurements.

However, even with the level of fidelity restricted by the spacing of the fiducial markers, the comparison between the experiment and the CPFEM results shown in Figure 6 exhibit good qualitative agreement. The results in Figure 6 are more resolved than the DIC results because of the higher resolution in the CPFEM mesh (Figure 2). One of the important aspects of the simulation results is that they capture both the character of the surface strains (i.e. the location of high and low strained regions) as well as the magnitudes of the strain components.

Another point to understand is that the comparison between the model and the experiments would be better characterized by directly comparing the displacements at the surface. Calculating the strain components from the displacement data is an extra step imposed on both the experiment and the model, where the displacements of the fiducial markers is the data directly measured by the DIC and is one of the direct outputs from the CPFEM model. However, when conducting the experimental micro-tension test we did not have an exact correlation between the location of the fiducial markers and the microstructure used to instantiate the CPFEM model. Therefore, comparing the location of the fiducial markers in the experiment and in the model was not possible. To further complicate this point, the serial section data was collected after the micro-tension test was already deformed, as the 3D-EBSD serial sectioning technique is a destructive process. This implies that the microstructure used to instantiate the CPFEM model was from the post-deformed micro-tension test, and therefore the model simulated a slightly different microstructure than the experiment. However, since the deformation was small (2.4\% axial true strain), the microstructure is not expected to have evolved much over that deformation, and we do not believe this made a large difference in the results. Some of the small differences seen in the comparison of Figure 4 and Figure 6 may be due to the different experimental and initial simulation microstructures, but it is not possible to determine the exact extent of this factor. New and emerging techniques such as High-Energy Diffraction Microscopy (HEDM) [40-42] utilizing high-energy $\mathrm{x}$-ray diffraction provides a means to nondestructively measure the initial internal three-dimensional microstructure as well as provide in situ information about the evolution of heterogeneous deformation below the specimen surface.

After verifying that our CPFEM model successfully captured the surface strains, we used the model to examine the sources of heterogeneity in the pure nickel micro-tensile specimen. We hope this will lead to a greater insight into heterogeneous plastic deformation to help address problems such as fatigue crack initiation, among others. One of the questions we investigated was the role of the sub-surface microstructure on the surface strain distribution. Using the serial sectioning capability in this manner is not always available or practical, especially when a statistically large data set is required over many specimens. The question then becomes: if the entire microstructure below the surface was an unknown quantity, then how different could our predictions of surface strain have been from our simulations if we likewise treated it as unknown. In this work we do not attempt to answer that entire question, but we do examine a subset of it where the grain morphology (size, shape and location) are known but the crystallographic orientations were randomly permuted in the subsurface grains. We ran 10 different simulations with the permuted sub-surface crystallographic orientations, and the surface strain distributions for one of these simulations are shown in Figure 8. Both the axial and transverse surface strains appear to match closely with the original simulation shown in Figure 6. This would seemingly indicate that the crystallographic orientations of the surface grains and/or their grain morphology are largely responsible for the surface strain distributions that develop during deformation. However, to more accurately examine the plots we have used a normalized difference expression through equation 4 and present those results in Figures $9 \& 10$. These figures show three different permuted simulations, and the areas of largest difference between the original and permuted simulations are apparent. Overall the differences that appeared small on Figure 8 can be as large as the macroscopic strain of the tensile deformation, indicating that the sub-surface crystallographic orientation does play a critical role in the measured surface strain distributions - both the magnitudes of those strains as well as their spatial locations. Again, this indicates that a complete understanding of the entire three-dimensional microstructure is a necessary component to truly simulating and capturing the details of the heterogeneous nature of deformation in this pure nickel material. Although we do not extend this analogy to changing the grain morphology of the sub surface grains, we expect that if we did change the grain size, shape or location of grains below the surface, that the differences in the permuted simulations would be even larger than those shown in Figures 10 \& 11. To emphasize this point in another manner: if we simulated a specimen where we only knew the crystallographic orientations of the surface grains because we could not or did not perform serial sectioning or HEDM experiments to determine the sub-surface grain morphologies and crystallographic orientations, then we would not expect to accurately capture the surface strains in the simulation.

For the lattice strain distribution experiments, we used the same crystal plasticity model to simulate an LSHR specimen that contained a large number of grains (approximately 50,000 grains). Figure 5 shows both the experimental and simulation results for the SPFs, where in both cases the lattice strain is largest along the loading direction for all three $\{h k l s\}$ shown. The match of the simulation to the experimental data in this figure is not a validation of the simulation, but rather another tool to further constrain the model. Once we had a calibrated simulation, we examined the difference in stress states between crystals in the interior of the simulation to those grains on the free surfaces.

Figure 3 shows the finite element representation of the microstructure used for the high-energy diffraction experiment. Clearly the regular arrangement of the grains and the artificial constraint that each grain was initially equiaxed and square in morphology is an unrealistic assumption. However, to capture distributions in lattice strain it is necessary to simulate a large number of crystals, which cannot be explicitly represented utilizing current computing resources. Although the individual grains in this simulation are not highly resolved (each grain is represented by 8 finite elements), we see from Figure 7 that both inter- and intra granular distributions of stress (the axial stress in Figure 7) develop during the simulation. 
To further characterize this heterogeneity in the deformation, we evaluated the distribution of stress for all the grains. Figure 11 shows this analysis for the $1080 \mathrm{MPa}$ axial-stress level. Despite the fact that the macroscopic loading conditions (uni-axial tension) are relatively simple, and that the specimen was only deformed to $3 \%$ axial true strain, we see significant heterogeneity in the stress state in Figure 11. This coaxiality of the stress states is defined by the usual dot product operation, and represents a scalar measure of the difference between two stress states. In Figure 11 we evaluated the stress coaxiality for the average stress state in each grain against the applied uni-axial stress state. If a grain experienced a perfect uni-axial stress state with the principal directions coincident with the sample loading direction, then the stress coaxiality would be zero. One of the striking conclusions of Figure 11 is that no single grain in the overall aggregate experiences uni-axial tension, even though the overall distribution of stress states must average to this condition. In addition, there is a significant difference between grains on the surface of the simulation as compared to grains in the interior. On average grains on the traction free surfaces have lower stress coaxiality with the applied stress state. This is not unexpected, as the grains at the surface satisfy the traction free condition with three stress components equal to zero. This applies a constraint to the surface grains that more closely matches the uni-axial stress state which doesn't exist in the interior of the simulation.

While Figure 11 provides great insight into the nature of the deformation, it only shows a distribution of the stress coaxiality. Another component of the heterogeneous nature of the deformation is the magnitude of the stress state in each grain. Figure 12 plots the normalized stress magnitude against the stress coaxiality, where ideal uni-axial tension is represented by $\Theta=0$, and a normalized stress magnitude of 1.0. Once again, the difference between the stress state of each grain and the ideal uniaxial stress state is apparent. In fact, there is a wide variation in stress magnitude from grain to grain, where some grains experience a normalized magnitude as high as 1.6, indicating the magnitude of their stress state is 1.6 times that of the applied uniaxial tension. Other grains experience a normalized stress magnitude of only 0.6 , indicating a large heterogeneity in the partitioning of the stress throughout the simulation. There is also a difference in the overall distribution of stress magnitude between the surface grains and the interior grains. Figure 12 shows that the stress states in the surface grains are closer to ideal uni-axial tension than grains in the interior. However, once again we see that no single grain in the entire aggregate experiences ideal uniaxial loading even though the body averages to a uni-axial stress state.

The simulation of the high energy diffraction experiment shows that significant heterogeneity exists in the stress state on the level of the individual grain, even in a simulation that does not explicitly attempt to accurately represent the morphology (grain size/shape) of individual crystals. This conclusion may be entirely obvious at the level of the micro-tension specimen, where the DIC measurements clearly show this heterogeneity. However, in a polycrystalline sample with a large number of grains and a relatively random distribution of crystallographic orientations, this level of heterogeneity is not necessarily intuitive. In addition, there is a significant difference in the distribution of stress states for surface grains as compared to grains in the bulk of the simulated volume. Therefore, even if we were to perform DIC on the surface of a large polycrystalline specimen, we would not capture the full distribution of strain. So while DIC is an important measurement tool that can be used for model validation, it is important to recognize the limitations to better understand the information it reveals.

\section{Conclusions}

\subsection{Multi-Crystal Simulation and Experiments}

- The density of the fiducial markers needs to be refined in future work to better capture the heterogeneous deformation we can use the simulations to guide their density in future work

- The CPFEM methodology presented here captures the heterogeneous deformation of the multi-crystal extremely well when the grain morphology and crystallographic orientations are used as inputs to the model

- While the crystallographic orientations of the surface grains appears to have the largest effect on the surface strain distributions, the sub-surface crystallographic orientations have a strong influence as well

- The full three-dimensional microstructure is needed to properly simulate a material specimen and resolve the heterogeneous nature of the deformation

\subsection{High Energy Diffraction Simulation and Experiments}

- High energy x-ray diffraction utilizing a synchrotron light source is a viable way to measure the lattice strains in situ.

- The distribution of lattice strains provides another point of comparison between the experiment and the simulation below the macroscopic length scale

- Significant deformation heterogeneity is predicted by the CPFEM simulation for the high-energy diffraction simulation even under relatively simple loading conditions

- A significant difference in the stress states exists for the grains at the surface of the simulation as compared to the grains in the interior of the simulation

- The use of DIC alone to understand the deformation in a polycrystalline material may be inadequate to fully characterize the deformation since it performs the measurement and analysis at a traction-free surface and does not necessarily provide the response of the entire specimen on average.

\section{Acknowledgements}

This work is based upon research conducted at the Cornell High Energy Synchrotron Source (CHESS) which is supported by the National Science Foundation under NSF award DMR-0225180.

\section{References}

1. R. Becker, "Analysis of Texture Evolution in Channel Die Compression-I. Effects of Grain Interaction", Acta metal mater 39 (6) (1991) 1211-1230

2. R. Becker, S. Panchanadeeswaran, "Effects of Grain Interactions on Deformation and Local Texture in Polycrystals", Acta metal. mater. 43 (7) (1995), 2701-2719

3. S. Panchanadeeswaran, R.D. Doherty, R. Becker, "Direct Observation of Orientation Change By Channel Die 
Compression of Polycrystalline Aluminum-Use of a Split Sample”, Acta mater. 44 (3) (1996) 1233-1262

4. A. Bhattacharyya, E. El-Danaf, S.R. Kalidindi, R.D. Doherty "Evolution of grain-scale microstructure during large strain simple compression of polycrystalline aluminum with quasicolumnar grains: OIM measurements and numerical simulations”, International Journal of Plasticity 17 (2001) 861883

5. E. Marin, P.R. Dawson "Elastoplastic finite element analysis of metal deformation using polycrystal constitutive models", Comp. Methods in Appl. Mech. and Eng, 165 (1998) 1-21

6. E. Marin, P.R. Dawson, "On modeling the elasto-viscoplastic response of metals using polycrystal plasticity", Comp. Methods in Appl. Mech. and Eng, 165 (1998) 23-41

7. N.R. Barton, J. Knap, A. Arsenlis, R. Becker, R.D. Hornung, D.R. Jefferson, "Embedded polycrystal plasticity and adaptive sampling”, Inter. J. Plasticity 24 (2008) 242-66

8. T.J. Turner, M.P. Miller, "Modeling the influence of material structure on deformation induced surface roughening in AA7050 thick plate", Journal of Engineering Materials and Technology, 129 (2007) 367-79

9. T.J. Turner, S.L. Semiatin, "Modeling large-strain deformation behaviour and neighborhood effects during hot working of a coarse-grain nickel-base superalloy", Modeling and Simulation in Materials Science and Engineering, 19 (2011), 1-25

10. J. Gayda,T.P. Gabb, P.T. Kantzos, "The Effect of Dual Microstructure Heat Treat on an Advanced Nickel-Base Disk Alloy”, (Paper presented at Superalloys 2004) 235-241

11. P.A. Shade, R. Wheeler, Y.S. Choi, M. Uchic, "A combined experimental and simulation study to examine lateral constraint effects on microcompression of single-slip oriented single crystals” Acta Mater, 57 (2009) 4580-87

12. M.D. Uchic, R. Wheeler, P.A. Shade, "Application of microsample testing to study fundamental aspects of plastic flow", Scripta Mater., 54 (2006) 759-64

13. R. Wheeler, P.A. Shade, M.D. Uchic, "Insights gained through image analysis during in-situ micromechanical experiments" , JOM In press

14. M.P. Miller, J.V. Bernier, J.S. Park, A. Kazimirov "Experimental measurement of lattice strain pole figures using synchrotron x rays”, Rev Sci Instrum 76 (11) (2005)

15. J.S. Park, P. Revesz, A. Kazimirov, M.P. Miller, “A methodology for measuring in situ lattice strain of bulk polycrystalline material under cyclic load”, Rev Sci Instrum 78 (2) (2007)

16. J.V. Bernier, M.P. Miller, J.S. Park, U. Lienert, "Quantitative stress analysis of recrystallized OFHC Cu subject to deformation in situ”, J Eng Mater Technol 130 (2) (2008)

17. J.V. Bernier, J.S. Park, A.L. Pilchak, M.G. Glavicic, M.P. Miller, "Measuring stress distributions in Ti-6Al-4V using synchrotron x-ray diffraction”, Metall Mater Trans, A 39 (13) (2008)

18. J.C. Schuren, M.P. Miller, A. Kazimirov, “A mechanical ettsing capability for measuring the microscale deformation behavior of structural materials”, Experimental Mechanics (2011) 1-19

19. M.P. Miller, J.S. Park, P.R. Dawson, T.S. Han, "Measuring and modeling distributions of stress state in deforming polycrystals", Acta Mater 56 (15) (2008)

20. P.R. Dawson, D.E. Boyce, S. MacEwen , R. Rogge, "On the influence of crystal elastic moduli on computed lattice strains in AA-5182 following plastic straining”, Materials Science and Engineering A313 (2001) 123-144

21. N.R. Barton, D.J. Benson, R. Becker, "Crystal level simulations using Eulerian finite element methods", Materials Processing and Design: Modeling, Simulation and Applications, NUMIFORM 2004 (College Park, MD: AIP) 712 (2004) 162429
22. N.R. Barton, D.J. Benson, R. Becker, "Crystal level continuum modelling of phase transformations: the a $\leftrightarrow$ e transformation in iron”, Modelling Simul. Mater. Sci. Eng 13 (2005) 707-31

23. W.F. Hosford The Mechanics of Crystals and Textured Polycrystals (New York, New York, Oxford Science Publications, 1993)

24. G.I. Taylor, "Plastic Strain in Metals", J. Inst. Met, 62 (1938) $307-24$

25. U.F. Kocks, "Laws for work-hardening and low-temperature creep”, ASME J. Eng. Mater. Tech, 98 (1976) 76-85

26. E. Voce, "The relationship between stress and strain for homogeneous deformation”, J. Inst. Met, 74 (1948) 537-62

27. K. Mathur, P.R. Dawson, "On modeling the development of crystallographic texture in bulk forming processes", Inter. J. Plasticity, 5 (1989) 67-94

28. D. Peirce, R.J. Asaro, A. Needleman, "Overview no. 21 An analysis of nonuniform and localized deformation in ductile single crystals”, Acta Metallurgica, 30 (6) (1982) 1087-1119

29. D. Peirce, R.J. Asaro, A. Needleman, "Material rate dependence and localized deformation in crystalline solids", Acta Metallurgica, 31 (12) (1983) 1951-1976

30. A. Needleman, R.J. Asaro, J. Lemonds, D. Peirce, "Finite element analysis of crystalline solids", Computer Methods in Applied Mechanics and Engineering, 52 (1-3) (1985) 689-708

31. R.J. Asaro, A. Needleman, "Overview no. 42 Texture development and strain hardening in rate dependent polycrystals”, Acta Metallurgica, 33 (6) (1985) 923-953

32. G.I. Taylor, C.F. Elam, "The distortion of an aluminum crystal during a tensile test”, Proc. Royal Soc. London A, A102 (1923) 643-667

33. G.I. Taylor, Analysis of Plastic Strain in a Cubic Crystal Timoshenko Anniversary Edition, MacMillan Press (New York) 218-224

34. A. Anderson, R. Cooper, R. Neely, A. Nichols, R. Sharp, B. Wallin, "Users manual for ALE3D - an arbitrary Lagrange/Eulerian 3D code system” (Technical Report UCRLMA-152204, Lawrence Livermore National Laboratory, 2003)

35. M.P. Miller, J.V. Bernier, J.S. Park, A. Kazimirov, "Experimental measurement of lattice strain pole figures using synchrotron x rays", Rev Sci Instrum 76 (11) (2005)

36. P. Dawson, D. Boyce, S. MacEwen, R. Rogge, "Residual Strains In HY100 Polycrystals: Comparisons of Experiments and Simulations”, Metallurgical and Materials TransactionsA, 31A (2000) 1543-1555

37. U.F. Kocks, C.N. Tome, H.R. Wenk, Texture and Anisotropy, (Cambridge University Press, 1998)

38. A. Yamaji, K. Sato K, "Distances for the solution of stress tensor inversion in relation to misfit angles that accompany the solutions”, Geophysics Journal International, 167 (2006) 933942

39. H. Ritz, P.R. Dawson, T. Marin, "Analyzing the orientation dependence of stresses in polycrystals using vertices of the single crystal yield surface and crystallographic fibers of orientation space", J Mech Phys Solid, 58 (2010) 54-72

40. R.M. Suter, D. Hennessy, C. Xiao, U. Lienert, "Forward Modeling method for microstructure reconstruction using x-ray diffraction microscopy: Single-crystal verification”, Rev. Sci. Instrum. 77 (2006)

41. U. Lienert, S.F. Li, C.M. Hefferan, J. Lind, R.M. Suter, J.V. Bernier, N.R. Barton, M.C. Brandes, M.J. Mills, M.P. Miller, B. Jakobsen, W. Pantleon, "High-Energy Diffraction Microscopy at the Advanced Photon Source: JOM 63:7 (2008) 70-77

42. U. Leinert, M.C. Brandes, J.V. Bernier, J. Weiss, S. Shastri, M.J. Mills, M.P. Miller, "In Situ single-grain peak profile measurements on Ti-7Al during tensile deformation", Materials Science and Engineering A, 524 (2009) 46-54 